\title{
Hippocampus and Retrosplenial Cortex Combine Path Integration Signals for Successful Navigation
}

\author{
Katherine R. Sherrill, ${ }^{1,2}$ Uğur M. Erdem, ${ }^{1}$ Robert S. Ross, ${ }^{1,2}$ Thackery I. Brown, ${ }^{1,2}$ Michael E. Hasselmo, ${ }^{1}$ \\ and Chantal E. Stern ${ }^{1,2}$ \\ ${ }^{1}$ Center for Memory and Brain, Boston University, Boston, Massachusetts 02215, and ${ }^{2}$ Athinoula A. Martinos Center for Biomedical Imaging, \\ Massachusetts General Hospital, Charlestown, Massachusetts 02129
}

\begin{abstract}
The current study used fMRI in humans to examine goal-directed navigation in an open field environment. We designed a task that required participants to encode survey-level spatial information and subsequently navigate to a goal location in either first person, third person, or survey perspectives. Critically, no distinguishing landmarks or goal location markers were present in the environment, thereby requiring participants to rely on path integration mechanisms for successful navigation. We focused our analysis on mechanisms related to navigation and mechanisms tracking linear distance to the goal location. Successful navigation required translation of encoded survey-level map information for orientation and implementation of a planned route to the goal. Our results demonstrate that successful first and third person navigation trials recruited the anterior hippocampus more than trials when the goal location was not successfully reached. When examining only successful trials, the retrosplenial and posterior parietal cortices were recruited for goal-directed navigation in both first person and third person perspectives. Unique to first person perspective navigation, the hippocampus was recruited to path integrate self-motion cues with location computations toward the goal location. Last, our results demonstrate that the hippocampus supports goal-directed navigation by actively tracking proximity to the goal throughout navigation. When using path integration mechanisms in first person and third person perspective navigation, the posterior hippocampus was more strongly recruited as participants approach the goal. These findings provide critical insight into the neural mechanisms by which we are able to use map-level representations of our environment to reach our navigational goals.
\end{abstract}

Key words: navigation; time; memory; parahippocampal cortex; posterior parietal cortex; optic flow

\section{Introduction}

Path integration uses self-motion cues to track adjustments in orientation and location (Wolbers et al., 2007). Research in rodents has demonstrated that hippocampal place cells can track current location related to a goal location (Johnson and Redish, 2007; Pfeiffer and Foster, 2013). In humans, several cortical regions in addition to the hippocampus guide navigation through the integration of spatial representations and self-motion cues to update goal-directed behavior (Aguirre and D'Esposito, 1999; Epstein, 2008; Save and Poucet, 2009; Vann et al., 2009; Whitlock et al., 2012). Studies in primates (Sato et al., 2006) and humans (Rosenbaum et al., 2004; Spiers and Maguire, 2006; Ekstrom and Bookheimer, 2007; Epstein et al., 2007; Rodriguez, 2010) suggest

Received May 1, 2013; revised 0ct. 23, 2013; accepted 0ct. 27, 2013.

Author contributions: K.R.S., U.M.E., R.S.R., T.I.B., M.E.H., and C.E.S. designed research; K.R.S. performed research; K.R.S., U.M.E., and T.I.B. analyzed data; K.R.S., U.M.E., R.S.R., T.I.B., M.E.H., and C.E.S. wrote the paper.

This work was conducted with the support of the Office of Naval Research (ONR) Multidisciplinary University Research Initiative (MURI) (N00014-10-1-0936). fMRI scanning was completed at the Athinoula A. Martinos Center for Biomedical Imaging (Charlestown, MA) which receives support from NCRR P41RR14075. We thank Henry Eve for assistance with analyzing statistical data, and Randall Newmark for assistance with fMRI data collection.

The authors declare no competing financial interests.

Correspondence should be addressed to Katherine Sherrill, M.A., Center for Memory and Brain, Boston University, 2 Cummington Mall, Boston, MA 02215. E-mail: krmck@bu.edu.

DOI:10.1523/JNEUROSCI.1825-13.2013

Copyright $\odot 2013$ the authors $\quad 0270-6474 / 13 / 3319304-10 \$ 15.00 / 0$ that the retrosplenial and posterior parietal cortices support the transformation of world coordinate-based spatial information into self-motion cues to guide movements from a ground-level perspective. Specifically, it has been suggested that the retrosplenial cortex (RSC) integrates route-based spatial information with self-motion cues (Wolbers and Buchel, 2005) and computes perceived heading (Baumann and Mattingley, 2010). These studies suggest that regions within the retrosplenial and posterior parietal cortices integrate current orientation with distance and direction toward the goal location as represented by the hippocampus.

Human spatial memory studies often target navigation from the first person perspective (FPP) in familiar, landmark-rich environments (Hartley et al., 2003; Wolbers and Büchel, 2005; Ekstrom and Bookheimer, 2007; Brown et al., 2010; Xu et al., 2010; Brown and Stern, 2013; Zhang and Ekstrom, 2013). The focus of this study was to examine path integration mechanisms for accurate navigation through the integration of orientation and selfmotion cues in the absence of landmark cues. When landmark information is not available, path integration can be used to build a metric representation of position. Place cells in the hippocampus provide spatial tuning through structured responses that code current position in an environment (O'Keefe and Dostrovsky, 1971; Ekstrom et al., 2003). Spatially tuned neurons of the hippocampus may track proximity to goal locations 
A

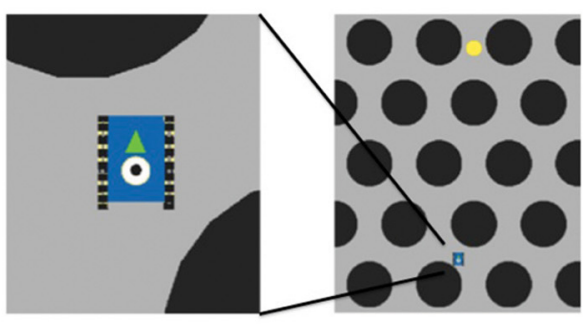

B

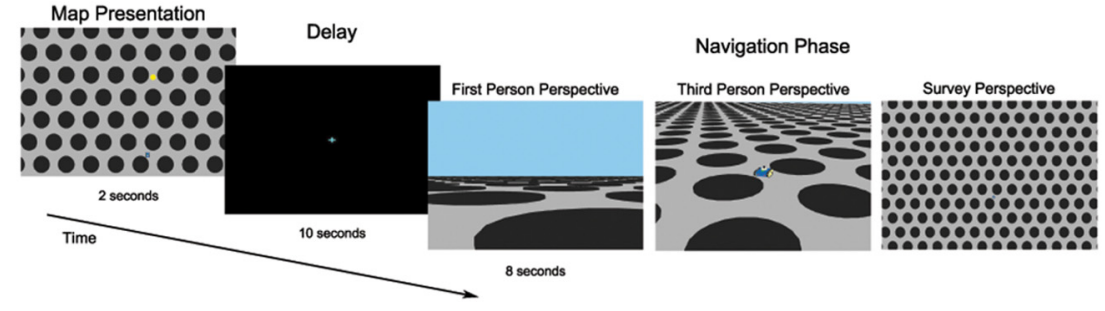

Figure 1. Task paradigm. $\boldsymbol{A}$, Survey perspective of the vehicle (blue) that was guided by participants to the goal location (yellow dot). Expanded view represents the vehicle; green arrow indicates orientation in the environment. $\boldsymbol{B}$, During the $2 \mathrm{~s}$ map presentation, participants were shown a survey representation of the environment with their start location, heading direction, and goal location clearly marked. Map presentation was followed by a 10 second delay, during which participants made no response. After the delay, there was an $8 \mathrm{~s}$ navigation phase requiring active navigation to the goal location in which movement occurred in one of three visual perspectives: FPP, TPP, or a survey perspective.

through navigational episodes (Johnson and Redish, 2007; Dupret et al., 2010; Viard et al., 2011). The spatial tuning of the hippocampus through integration of current location and goal proximity provides essential mapping mechanisms required for path integration.

The present study provides novel insight into the encoding of survey-level spatial information required for ground-level, goaldirected navigation and the integration of these encoded spatial representations with path integration mechanisms for successful navigation. On each trial, participants viewed a map of a landmark-deprived environment indicating the start and goal locations and then used these survey-level spatial representations to actively navigate the environment. Navigation occurred from first person, third person, or survey perspectives. We predicted that the posterior parietal and retrosplenial cortices would encode survey-level representations of the environment and recruit these spatial representations for active, ground-level navigation. We predicted that the hippocampus would be uniquely recruited for FPP navigation when monitoring selfmotion would be integral to navigation success. Critically, we predicted the hippocampus would be important for tracking distance to the goal location during ground-level navigation relying on the integration of survey representations and self-motion cues.

\section{Materials and Methods \\ Participants}

Thirty-four participants were recruited for this study from the Boston University community. All participants were right-handed and had selfreported experience playing video games. Written informed consent was obtained from each participant before enrollment in accordance with the experimental protocol approved by both the Partners Human Research Committee and the Boston University Charles River Campus Institutional Review Board.

Four participants were eliminated from the final analysis because of excessive motion during fMRI scanning and six additional participants were eliminated because of technical issues during the scanning sessions. Twenty-three participants were included in the final parametric data analysis (mean $\pm \mathrm{SD}$ age $22.461 \pm 3.49 ; 13$ males, 10 females). A subset of participants was included in a whole-brain analysis of navigators who scored at least $50 \%$ correct on all trials in each perspective (18 participants; age $22.801 \pm 3.50$; 12 males, 6 females). The number of correct trials was not large enough to include participants with less than $50 \%$ correct trials in each perspective in the whole-brain analysis. However, participants who did not score at least $50 \%$ correct on all trials in each perspective but had little movement in the scanner were included in the linear regression analysis (parametric).

\section{Virtual environment}

We developed a navigation task in which participants encoded a start and goal location from a survey-level map perspective and subsequently translated this spatial representation into accurate, goal-directed navigation in a first person perspective (FPP), third person perspective (TPP), or Survey perspective. Critically, the environment contained no distinguishing landmarks, distal cues, or goal location markers. Panda3D Software (Entertainment Technology Center, Carnegie Mellon University) was used to create a virtual environment consisting of an open field extending in all directions toward the horizon and sky (Fig. 1B). Within the virtual environment, one virtual unit represented 1 meter. Short circular columns (radius six virtual units, height 0.15 virtual units) were placed upon the floor of the open field environment in a $60^{\circ}$ hexagonal pattern. While moving through the virtual space, a participant could not traverse across any column. This prevented participants from moving directly to the goal location in a straight line, encouraging active computation and maintenance of orientation as their route arced around the columns.

We varied the initial heading direction across trials (facing north, east, west, south). We also varied the bearing of the goal location relative to the start location $\left(30^{\circ}, 60^{\circ}, 90^{\circ}, 120^{\circ}\right.$, or $150^{\circ}$ angles). Heading direction and goal location bearing at the start location were counterbalanced across trial conditions and runs. Participants were informed that their heading direction at the start of the navigation phase would always be facing the cardinal direction indicated by an arrow on the map presentation (Fig. 1A).

Participants navigated through the environment using a button response box. Movement was simulated using three button responses corresponding to the left, forward, and right directions. Participants could not navigate in a reverse direction. Button presses could occur simultaneously (i.e., left and forward), allowing for a smooth range of simulated motion. Navigation occurred in one of three visual perspectives: FPP, TPP, or a Survey perspective (Fig. $1 B$ ). In all three visual perspectives, movement speed was held constant at 5 virtual units per hour, the equivalent of a 5 kilometer per hour walking speed. In the FPP, the participant's perspective was set at a height of 2 virtual units to represent a 2 meter tall person walking through the virtual environment. The field of view during FPP navigation was restricted to the scene in front of the participant, consistent with the definition of FPP. Optic flow was representative of what a person walking through the environment would experience. In the TPP, the participant's perspective was set at a height of 7 virtual units, and a vehicle was guided by the participant to the goal location while the participant remained stationary in the environment (i.e., the camera did not translate with the vehicle). The field of view from the TPP encompassed a larger portion of the environment. During TPP-guided navigation, the vehicle always remained at the center of the participant's field of view. In the Survey perspective, the participant steered a vehicle to the goal location from a fixed, survey-level perspective looking directly down at the $0,0,0$ coordinate (Fig. $1 B$ ).

\section{Experimental training}

Prescan training. One day before scanning, participants became familiarized with the button box controls and the three different navigation 
perspectives of the virtual environment (FPP, TPP, and Survey perspective). Participants spent at least $12 \mathrm{~min}$ practicing navigating in each visual perspective with no goal location present. Participants then completed five practice runs with $50 \%$ accuracy to ensure their ease with the navigational controls and their understanding of the task design. Three practice runs included trials with the navigation phase unique to one perspective (i.e., all four trials in one run had FPP navigation phases). Last, two practice runs were composed of 12 trials randomly counterbalanced to include navigation phases in all three perspectives (FPP, TPP, and Survey). Participants had to complete the last two practice runs with at least $60 \%$ accuracy to take part in fMRI scanning.

Experimental testing during $f M R I$ scanning. Scanning data were collected the day after training. Participants were given a practice run to refamiliarize themselves with the task and keyboard controls before being placed in the scanner. During scanning, participants performed 10 runs composed of 12 trials per run. Each trial consisted of map presentation, delay, and navigation phases, followed by an intertrial interval. Trials of the FPP, TPP, and Survey perspective conditions were presented in an interleaved, randomized order. During the $2 \mathrm{~s}$ map presentation, participants were shown a survey representation of the environment with their start location, heading direction, and goal location clearly marked. The $2 \mathrm{~s}$ duration of the map presentation phase discouraged participants from merely counting columns to navigate to the goal location. Because of the short duration of the map presentation, route planning was based on orientation from the start location to the goal location. The map presentation phase was followed by a $10 \mathrm{~s}$ delay, during which participants made no response. After the delay, there was an $8 \mathrm{~s}$ navigation phase requiring active navigation to the goal location. Participants were instructed to navigate to the precise location where they thought the encoded goal was located. The goal location was not visible during the navigation phase, and no feedback was given as to whether the participant successfully reached the goal location. A trial was considered correct if participants' trajectories during the navigation phase came within a radius of 3 virtual units from the goal location. The distance between the start location and goal location was on average $25.78 \pm 1.61$ (SD) virtual units across all trials. Therefore, 3 virtual units correspond to $11.6 \%$ of the average distance between the start and goal location. Critically, no distinguishing landmarks, distal cues, or goal location markers were present in the environment. This required participants to merge selfmotion cues from optic flow with their planned route during groundlevel navigation. Participants did not know trial type (FPP, TPP, or Survey perspective navigation) until the start of the navigation phase. The order of the trials was counterbalanced across runs, and the order of runs was randomized across participants. There were 40 trials per experimental condition.

\section{Image acquisition}

Images were acquired at the Athinoula A. Martinos Center for Biomedical Imaging, Massachusetts General Hospital (Charlestown, Massachusetts) using a 3 Tesla Siemens MAGNETOM TrioTim scanner with a 32-channel Tim Matrix head coil. A high-resolution T1-weighted MPRAGE structural scan was acquired using Generalized Autocalibrating Partially Parallel Acquisitions (GRAPPA) $(\mathrm{TR}=2530 \mathrm{~ms}$; $\mathrm{TE}=3.31 \mathrm{~ms}$; flip angle $=7^{\circ}$; slices $=176$; resolution $=1 \mathrm{~mm}$ isotropic). T2* -weighted BOLD images were acquired using an Echo Planar Imaging sequence $\left(\mathrm{TR}=2000 \mathrm{~ms} ; \mathrm{TE}=30 \mathrm{~ms}\right.$; flip angle $=85^{\circ}$; slices $=33$, resolution $=$ $3.4 \times 3.4 \times 3.4 \mathrm{~mm}$, interslice gap of $0.5 \mathrm{~mm}$ ). Functional image slices were aligned parallel to the long axis of the hippocampus.

\section{fMRI preprocessing}

Functional imaging data were preprocessed and statically analyzed using the SPM8 software package (Statistical Parametric Mapping, Wellcome Department of Cognitive Neurology, London). All BOLD images were first reoriented so the origin (i.e., coordinate $\mathrm{x} \mathrm{y} \mathrm{z}=[0,0,0]$ ) was at the anterior commissure. The images were then corrected for differences in slice timing and were realigned to the first image collected within a series. Motion correction was conducted next and included realigning and unwarping the BOLD images to the first image in the series to correct for image distortions caused by susceptibility-by-movement interactions (Andersson et al., 2001).
Realignment was estimated using second-degree B-spline interpolation with no wrapping, whereas unwarp reslicing was done using fourth-degree $\mathrm{B}$-spline interpolation with no wrapping. The high-resolution structural image was then coregistered to the mean BOLD image created during motion correction and segmented into white and gray matter images. The biascorrected structural image and coregistered BOLD images were spatially normalized into standard MNI space using the Diffeomorphic Anatomical Registration Through Exponentiated Lie algebra (DARTEL) algorithm (Ashburner, 2007) for improved intersubject registration. BOLD images were resampled during normalization to $2 \mathrm{~mm}^{3}$ isotropic voxels and smoothed using a $6 \mathrm{~mm}$ full-width at halfmaximum Gaussian kernel. The normalized structural images of all 23 participants were averaged after normalization for displaying overlays of functional data.

\section{Behavioral analyses}

Behavioral performance. To compare overall performance between the FPP, TPP, and Survey perspective experimental conditions, an ANOVA was run comparing accuracy performance. Individual trials were considered correct if participants' trajectories during the navigation phase came within a radius of 3 virtual units from the goal location. Behavioral analyses were completed using PASW Statistics 18 (SPSS).

\section{fMRI analysis}

Whole-brain analyses. To model the data, separate regressors were created for the map presentation, delay, navigation phase, and intertrial interval for each condition (FPP, TPP, and Survey). Correct trials and incorrect trials were modeled separately for a total of 24 regressors. The six motion parameters calculated during motion correction were added to the model as additional covariates of no interest. Regressors from the task were constructed as a series of square waves or "boxcars." Boxcar onsets were defined by the onset of each event and extended for the duration of the event ( $2 \mathrm{~s}$ for map presentation, $10 \mathrm{~s}$ for the delay, $8 \mathrm{~s}$ for the navigation phase, and a $4-12 \mathrm{~s}$ variable duration for the intertrial interval). These parameters were convolved with the canonical hemodynamic response function in SPM8.

The model was then analyzed using the general linear model approach. Participant-specific parameter estimates pertaining to each regressor were calculated. The $t$-contrasts between the FPP, TPP, and Survey perspectives for the two task components of interest (map presentation and navigation phase) were constructed for each participant. Groupaveraged statistical parametric maps were created by entering the FPP, TPP, and Survey conditions (FPP > Survey; TPP > Survey; FPP > TPP; TPP $>$ FPP) contrast images from each participant into a one-sample $t$ test using participant as a random factor.

For each analysis, a voxelwise statistical threshold of $p<0.01$ was applied to the whole-brain contrast maps. To correct for multiple comparisons, we applied a cluster-extent threshold technique. The AlphaSim program in the AFNI software package (http://afni.nimh.nih.gov/afni/) was used to conduct a 10,000 iteration, $6 \mathrm{~mm}$ autocorrelation Monte Carlo simulation analysis on voxels within the group functional brain space using the ResMS header file (173,458 voxels). From this analysis, a minimum voxel extent of 144 was determined to maintain a family-wise error rate of $p<0.01$.

Correct versus incorrect navigation trials. We examined successful versus unsuccessful ground-level navigation by comparing correct trials versus incorrect trials. Parameter estimates of the FPP and TPP successful navigation trials were combined and contrasted against the combined parameter estimates of FPP and TPP navigation trials where participants were unsuccessful in reaching the goal location. The contrast images were then entered into a one-sample $t$ test using participant as a random factor. There were not enough error trials to analyze FPP and TPP separately, yet by combining the two conditions we could more broadly examine navigational accuracy.

Parametric modulation of linear distance to goal location. To examine how successful ground-level navigation integrated with spatial representations encoded at the survey-level, we conducted a parametric modulation analysis testing whether hippocampal activation tracks linear distance to the goal location from moment to moment during the navi- 
gation phase. The parametric $\mathrm{AMRI}$ data analysis was conducted using a targeted ROI approach. We predicted that the hippocampus would support goal-directed navigation by maintaining a guidance system to track linear distance to the goal location. To test this hypothesis, we created an anatomical ROI mask with a dilation of zero from the anatomical boundaries of the left and right hemisphere hippocampi using the Wake Forest University Pick-Atlas (Maldjian et al., 2003) available for SPM.

For the parametric analysis, the models from the previous analyses were modified into a new model such that the FPP and TPP navigation regressors were defined by stick functions sampled at each second of the trials. Parametric modulators for these regressors contained the normalized distance-to-goal values corresponding to each of these time points (Spiers and Maguire, 2007). Distance to the goal location was calculated as the shortest linear distance between the participant's current location and the goal location $(d)$. We rescaled the distance to goal to between 0 and 1 , with a value of 1 indicating the participant was at the goal location and a value of 0 reflecting the farthest distance from the goal location on a given trial $\left(1-d / d_{\max }\right.$ where $d_{\max }$ is the absolute distance from start location to goal location).

Separate one-sample $t$ tests for both the FPP and TPP conditions were conducted within our ROI volume. Similar to the whole-brain analysis, we applied a voxelwise statistical threshold of $p<0.01$ to the contrast maps. From a 10,000 iteration, $6 \mathrm{~mm}$ autocorrelation Monte Carlo simulation of the ROI volume (1878 voxels) in AlphaSim, a minimum voxel extent of 32 was determined to maintain a family-wise error rate of $p<0.01$.

To examine the relative influence of time and distance to goal on hippocampal activations during ground-level navigation, we conducted a second parametric modulation analysis testing the strength of the relationship between hippocampal activity and time during the navigation phase. The parametric fMRI data analysis was conducted using the same ROI approach as the distance to goal parametric analysis with FPP and TPP navigation regressors defined by stick functions sampled at each second of the trials. For the time analysis, the parametric modulators for these regressors were modified to contain time values (e.g., 1, 2, 3, etc.) corresponding to each second of the navigation phase.

Separate one-sample $t$ tests for both the FPP and TPP conditions were conducted within our ROI volume. We applied a voxelwise statistical threshold of $p<0.01$ to the contrast maps. To compare the relative effect sizes of distance and time on hippocampal activity, parameter estimates were extracted from $5 \mathrm{~mm}$ spheres centered on peak coordinates in the hippocampus for FPP and TPP during the navigation phase. A paired sample $t$ test between extracted parameter estimates for the distance to goal and time analyses was conducted using PASW Statistics 18 (SPSS).

Successful perspective specific analysis. One-sample $t$ tests were constructed of contrast images comparing the FPP and TPP conditions with the Survey perspective (FPP $>$ Survey; TPP $>$ Survey). The Survey perspective presented a bird's eye view of the entire environment and was visually identical to the map presentation. When navigating in the Survey perspective, participants had to simply navigate the vehicle to match the map information maintained in visual short-term memory. By comparing the FPP and TPP conditions with the Survey perspective during the navigation phase, we controlled for task components, such as motor responses, isolating activity related to integrating map information into ground-level navigation and processing self-motion from optic flow. FPP $>$ Survey and TPP $>$ Survey contrasts were constructed for both the map presentation and navigation phases of the experimental task. We also directly contrasted activity for FPP and TPP (FPP > TPP; TPP > FPP) for the map presentation and navigation phases of the experimental task.

Parameter estimates were extracted from $5 \mathrm{~mm}$ spheres centered on peak coordinates in our ROIs (hippocampus, retrosplenial cortex, posterior parietal cortex, and parahippocampal cortex) for contrasts between FPP, TPP, and Survey perspectives during the map presentation and navigation phases. Paired sample $t$ tests between conditions for the map presentation and navigation phases were conducted using PASW Statistics 18 (SPSS).

\section{Viewpoint Accuracy}

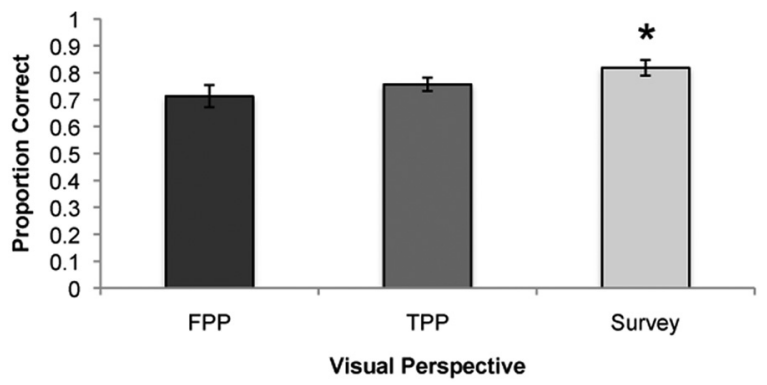

Figure 2. Scanning day behavioral performance. Error bars indicate SEM. *Significant difference. The chart represents the proportion of correct trials for FPP, TPP, or a survey perspective.
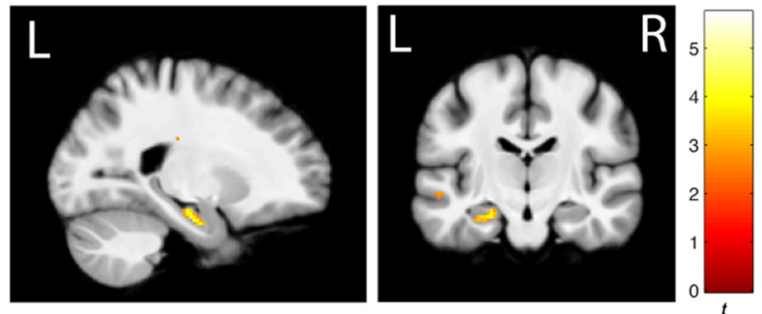

Figure 3. Successful FPP and TPP navigation recruits the anterior hippocampus. The wholebrain analyses image has a statistical threshold of $p<0.01$ corrected for multiple comparisons with a voxel extent of 144

\section{Results}

\section{Behavioral data}

We examined navigation performance to determine whether there were any differences in accuracy when navigating from FPP, TPP, and Survey perspectives. Participants reached the goal with precision in the FPP in $71.25 \%$ of the trials (SEM 4.09), the TPP in $75.69 \%$ of the trials (SEM 2.53), and the Survey perspective in $81.81 \%$ of the trials (SEM 2.91) (Fig. 2). A repeated-measures General Linear Model revealed a significant main effect of Perspective $\left(F_{(1,17)}=\right.$ $858.41, p<0.001)$. Follow-up $t$ tests revealed that the main effect of Perspective was driven by the Survey perspective, which had more correct trials than the FPP $(p=0.017)$ and the TPP $(p=0.044)$. Importantly, no significant differences in percentage correct were found using paired sample $t$ tests between the FPP and TPP conditions during the navigation phase $(p<0.178)$, indicating that the two ground-level conditions were completed with comparable accuracy. Participants navigated to the goal location in $6.32 \pm 0.06$ (SD) seconds on average across all trials.

\section{fMRI data}

\section{Correct versus incorrect trials $f M R I$ analysis}

To examine brain regions contributing to successful navigation, we contrasted successful FPP and TPP navigation trials with navigation trials in which the participant was unsuccessful in reaching the goal location. Our whole-brain analysis demonstrated that the anterior hippocampus was active for trials in which the participant successfully navigated to the goal location (Fig. 3; Table 1). This finding suggests that the differential activity for successful navigation involves computation in the anterior hippocampus.

\section{Parametric analysis of proximity to goal}

A primary goal of the experiment was to test whether the hippocampus actively tracks goal proximity (linear distance to goal location). Because the columns prevented direct (straight line) navigation to 
Table 1. Brain regions significantly activated for FPP and TPP navigation phases in which participants successfully navigated to the goal location ${ }^{a}$

\begin{tabular}{|c|c|c|c|c|c|c|c|}
\hline \multirow[b]{2}{*}{ Contrast } & \multirow[b]{2}{*}{ Area } & \multirow[b]{2}{*}{$K_{E}$} & \multicolumn{2}{|l|}{ Left } & \multirow[b]{2}{*}{$K_{E}$} & \multicolumn{2}{|c|}{ Right } \\
\hline & & & $\mathrm{T}$ & MNI $x, y, z$ & & $\mathrm{~T}$ & MNI $x, y, z$ \\
\hline Successful $>$ Unsuccessful & Hippocampus (head) & 178 & 4.25 & $-26,-12,-22$ & & & \\
\hline \multirow[t]{4}{*}{ FPP and TPP navigation phases } & Precuneus & 419 & 4.20 & $-12,-56,34$ & 419 & 3.26 & $4,-54,26$ \\
\hline & Superior frontal gyrus & 312 & 4.23 & $-16,-46,44$ & & & \\
\hline & Angular gyrus & 255 & 3.59 & $-46,-60,24$ & & & \\
\hline & Middle temporal gyrus & 398 & 5.46 & $-56,6,-24$ & 222 & 5.73 & $64,-2,-18$ \\
\hline
\end{tabular}

${ }^{a} \mathrm{MNI}$ coordinates reflect cluster-center voxels. T-values reflect a statistical threshold of $p<0.01$. Activation clusters survived cluster-threshold correction for multiple comparisons to $p<0.01$ with a minimum cluster size of 144 .

the goal, participants needed to integrate visual motion cues to accurately monitor the spatial relationship of their current location and the goal location while circumnavigating the obstacles. The left and right posterior hippocampus was modulated with the participants' distance to the goal location at time points sampled throughout the FPP navigation phase (Fig. 4A). Right posterior hippocampal activity was also modulated with linear distance to the goal location during the TPP navigation phase, although this activation was significant at a lower cluster extent threshold ( $p<0.05$ cluster significance). For a summary of brain regions activated at the whole-brain level for the parametric analysis of proximity to the goal location in the FPP, see Table 2.

To further characterize the role of hippocampal activation during the navigation phase, we examined activations associated with the progression of time across the navigation phase. The left posterior hippocampus was modulated with time during the FPP navigation phase $\left(t_{(23)}=3.29\right)$. Time was not correlated with navigation activity in the TPP, even at a lower statistical threshold of $p<0.05$. Parameter estimate extractions demonstrated that left posterior hippocampal beta weights were significantly greater for the distance to goal analysis than the time analysis $\left(t_{(23)}=2.118, p=0.046\right.$ ) (Fig. $4 B$ ). Furthermore, although activity in the right posterior hippocampus was significantly modulated by distance to goal, the linear effects of time did not reach statistical significance in this region. Together, these results indicate that distance to goal has a stronger influence on posterior hippocampal activity than a measure of time.

Navigation requiring path integration mechanisms to update perceived location and orientation toward a goal location

We examined activity during the navigation phase in which participants were at a ground-level (FPP and TPP) perspective and retrieved survey-level spatial information to successfully navigate to the goal location. The hippocampus, retrosplenial cortex, and posterior parietal cortex were more strongly recruited for FPP than Survey perspective during the navigation phase (Fig. 5A; Table 3). The retrosplenial cortex and parahippocampal cortex were more strongly activated for TPP than Survey perspective during the navigation phase (Fig. 5B; Table 3). Direct contrasts of the FPP and TPP conditions revealed a difference in the relative recruitment of retrosplenial and parahippocampal cortices during these two navigational perspectives. The retrosplenial cortex was active along with other brain regions when contrasting FPP against TPP navigation (Table 3). When contrasting TPP against FPP, the parahippocampal cortex had significantly greater activation. Although the retrosplenial and parahippocampal cortices were both active when FPP and TPP were contrasted against the Sur- vey perspective, retrosplenial function was more strongly recruited in the FPP, and navigation in the TPP more strongly recruited the parahippocampal cortex.

\section{Encoding of survey-level spatial information required for goal-directed navigation}

Successful navigation in the task requires that participants encode the start location, initial orientation, and goal location during the map presentation phase. Importantly, participants were unaware during the map presentation phase of the visual perspective in which they would subsequently be tested during the navigation phase. Regions activated during the map presentation support the encoding of survey-level spatial information required for successful navigation to the goal location. Therefore, comparison of map phase activation corresponding to correct FPP and TPP navigation trials against the map presentation for correct Survey navigation trials was analogous to subsequent memory paradigms. Several brain regions were commonly activated for map encoding on successful subsequent FPP and TPP navigation trials (Table 4). These regions included the retrosplenial cortex, posterior parietal cortex, and parahippocampal cortex (Fig. 6A,B). These common activations during map presentation for successful FPP and TPP trials relative to correct Survey trials may facilitate encoding of map information into a representation useful specifically for successful ground-level navigation. The results demonstrate that activation in the bilateral hippocampus at map presentation contributed to successful FPP navigation to the goal location (Fig. 6A).

Direct contrast of map presentation for successful FPP versus successful TPP trials $(\mathrm{FPP}>$ TPP; TPP $>$ FPP) revealed activation differences specific to the FPP. The brain regions active when contrasting encoding-related activity during map presentation of successful FPP navigation trials against the map presentation 
Table 2. Brain regions exhibiting significant activity modulated with the participants' distance to the goal location at time points sampled throughout the FPP navigation phase $^{a}$

\begin{tabular}{|c|c|c|c|c|c|c|c|}
\hline \multirow[b]{2}{*}{ Contrast } & \multirow[b]{2}{*}{ Area } & \multirow[b]{2}{*}{$\mathrm{K}_{\mathrm{E}}$} & \multicolumn{2}{|l|}{ Left } & \multirow[b]{2}{*}{$K_{E}$} & \multicolumn{2}{|l|}{ Right } \\
\hline & & & $\mathrm{T}$ & MNI $x, y, z$ & & $\mathrm{~T}$ & MNI $x, y, z$ \\
\hline \multirow{12}{*}{ FPP distance analysis } & Hippocampus (tail) & 18,369 & 3.36 & $-18,-40,4$ & 18,369 & 3.44 & $24,-40,4$ \\
\hline & Precuneus & & 3.81 & $-4,-72,52$ & 18,369 & 5.81 & $4,-62,60$ \\
\hline & Superior parietal lobule & & 4.10 & $-18,-70,60$ & & 5.44 & $20,-72,58$ \\
\hline & Dorsal lateral prefrontal cortex & 372 & 3.50 & $-38,34,32$ & & 3.96 & $42,34,36$ \\
\hline & Caudate (dorsal) & 18,369 & 2.56 & $12,4,12$ & & 2.93 & $-12,-2,16$ \\
\hline & Superior marginal gyrus & & 6.05 & $-42,-54,46$ & & 7.10 & $44,-58,48$ \\
\hline & Superior frontal gyrus & & 3.64 & $-4,24,40$ & & 3.55 & $8,24,46$ \\
\hline & Middle temporal gyrus & & & & 485 & 3.69 & $62,-50,-8$ \\
\hline & Insula & & 3.20 & $-42,10,6$ & 18,369 & 3.90 & $44,16,-4$ \\
\hline & Cuneus & & 2.93 & $-4,-80,26$ & & 2.79 & $4,-72,20$ \\
\hline & Pons & 276 & 4.41 & $-2,-30,-34$ & 276 & 4.70 & $1,-30,-34$ \\
\hline & Cerebellum & 157 & 3.76 & $-20,-28,-34$ & & 4.00 & $24,-30,-30$ \\
\hline
\end{tabular}

${ }^{a} \mathrm{MNI}$ coordinates reflect cluster-center voxels. T-values reflect a statistical threshold of $p<0.01$. Activation clusters survived cluster-threshold correction for multiple comparisons to $p<0.01$ with a minimum cluster size of 144 .
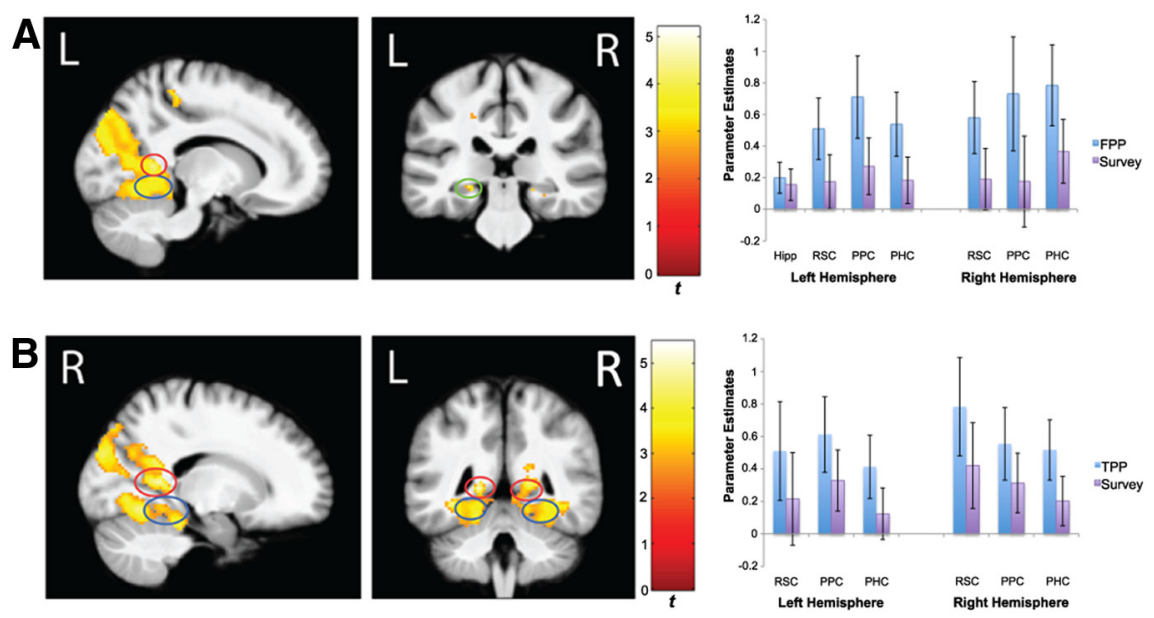

Figure 5. Activations for navigation trial phase. Both whole-brain analyses images have a statistical threshold of $p<0.01$ corrected for multiple comparisons with a voxel extent of 144. Green circles represent hippocampal (Hipp) activations; red circles represent retrosplenial cortex (RSC) activations; purple circles represent posterior parietal cortex (PPC) activations; blue circles represent parahippocampal cortex (PHC) activations. A, Whole-brain image of activity significantly greater for FPP navigation against Survey navigation (FPP $>$ Survey). Parameter estimate extractions from ROls are plotted on the right. Error bars indicate SEM. $\boldsymbol{B}$, Whole-brain image of activity significantly greater for TPP navigation than Survey navigation (TPP $>$ Survey). Parameter estimate extractions from ROls are plotted on the right. Error bars indicate SEM.

phase of successful TPP navigation trials were the retrosplenial cortex and parahippocampal cortex (Table 4). There were no significant TPP greater than FPP differences during map presentation.

\section{Discussion}

We used a task that required the encoding of survey-level map representations of the environment and subsequent navigation to a goal location in the FPP, TPP, or Survey perspective. Critically, no landmarks or distal cues were present in the environment. Our study found four main results: (1) anterior hippocampus activation when participants successfully navigated to the goal location; (2) a novel demonstration that the posterior hippocampus plays a role in coding proximity to a goal location during active navigation; (3) the retrosplenial cortex and posterior parietal cortex were recruited for successful navigation in both the FPP and TPP; and (4) path integration using selfmotion cues and orientation toward the goal location during successful FPP navigation recruited the hippocampus.
Successful navigation recruits the anterior hippocampus

Rodent models of navigation theorize place cell representations of location drive expectations of reward for goal locations (Foster et al., 2000; Johnson and Redish, 2007). In particular, the rodent ventral hippocampus, the analog for the human anterior hippocampus, has been associated with context and reward processing (Moser and Moser, 1998; Ferbinteanu and McDonald, 2001; Fanselow and Dong, 2010; Royer et al., 2010), suggesting that goal locations may be represented by the anterior hippocampus. A recent fMRI study demonstrated that the anterior hippocampus activates during spatial planning and the relative distance between the start location and goal (Viard et al., 2011). In the present study, FPP and TPP successful navigation trials recruited the anterior hippocampus more than trials when the goal location was not precisely reached. The results demonstrate that accurate ground-level navigation to the goal location recruits the anterior hippocampus. We suggest this recruitment may serve to successfully integrate orientation with a planned route.

\section{The posterior hippocampus tracks linear distance to the goal location}

Spatial coding for goal proximity within the hippocampus is relatively novel in studies of human navigation. Previous studies have suggested goal-directed navigation relies upon the integration of spatial representations from the hippocampus with goalrelated information from regions outside of the medial temporal lobe (MTL) (Spiers and Maguire, 2007). However, the presence of place-goal conjunctive cells in the human hippocampus, which increased their firing rate when a specific goal was viewed from a specific location, may be indicative of a hippocampal role in associating goal-related contextual inputs with place (Ekstrom et al., 2003). Yet, little is known about whether the hippocampus supports a mechanism for actively tracking progress to goal locations. A recent computational model suggests that a reward signal propagates through a place cell map of the environment originating from goal locations (Erdem and Hasselmo, 2012). Place cells 
Table 3. Brain regions exhibiting significant activity from paired $t$ test during the navigation phase ${ }^{a}$

\begin{tabular}{|c|c|c|c|c|c|c|c|}
\hline \multirow[b]{2}{*}{ Contrast } & \multirow[b]{2}{*}{ Area } & \multirow[b]{2}{*}{$K_{E}$} & \multicolumn{2}{|l|}{ Left } & \multirow[b]{2}{*}{$K_{E}$} & \multicolumn{2}{|l|}{ Right } \\
\hline & & & $T$ & MNI $x, y, z$ & & $T$ & MNI $x, y, z$ \\
\hline \multirow[t]{4}{*}{ FPP $>$ Survey } & Hippocampus (body) & 10,192 & 4.09 & $-24,-30,-8$ & & & \\
\hline & Parahippocampal cortex & & 4.57 & $-18,-44,-4$ & & 4.65 & $14,-46,-10$ \\
\hline & Cuneus & & 4.28 & $-4,-78,20$ & & 4.10 & $10,-76,20$ \\
\hline & Fusiform gyrus & & 4.41 & $-18,-56,-12$ & & 4.31 & $12,-52,-8$ \\
\hline \multirow[t]{6}{*}{ TPP $>$ Survey } & Retrosplenial cortex & 10,193 & 3.35 & $-12,-52,6$ & 10,193 & 5.23 & $16,-52,8$ \\
\hline & Precuneus & & 4.43 & $-8,-84,42$ & & 3.82 & $22,-76,46$ \\
\hline & Parahippocampal cortex & & 3.26 & $-22,-40,-12$ & & 3.75 & $28,-38,-12$ \\
\hline & Cuneus & & 4.98 & $-24,-90,28$ & & 4.53 & $20,-90,26$ \\
\hline & Lingual gyrus & & 3.87 & $-8,-68,-6$ & & 5.22 & $10,-66,-6$ \\
\hline & Fusiform gyrus & & 3.82 & $-22,-64,-10$ & & 4.91 & $24,-64,-14$ \\
\hline \multirow{2}{*}{$\mathrm{FPP}>\mathrm{TPP}$} & Posterior cingulate gyrus & & & & 200 & 3.88 & $10,-14,40$ \\
\hline & Postcentral gyrus & 199 & 2.63 & $-12,-40,46$ & & & \\
\hline \multirow[t]{7}{*}{$\mathrm{TPP}>\mathrm{FPP}$} & Parahippocampal cortex & & & & 1288 & 4.86 & $34,-34,-18$ \\
\hline & Superior parietal lobule & 574 & 3.93 & $-42,-36,52$ & & & \\
\hline & Dorsal lateral prefrontal cortex & & 4.48 & $-56,4,44$ & 340 & 3.05 & $52,34,16$ \\
\hline & Lateral occipital gyrus & 1744 & 6.03 & $-42,-66-16$ & 1288 & 4.77 & $40,-64,-18$ \\
\hline & Tempo-occipital gyrus & & 3.73 & $-34,-40,-24$ & & 4.76 & $34,-40,-24$ \\
\hline & Angular gyrus & & & & 336 & 4.79 & $30,-70,24$ \\
\hline & Postcentral gyrus & 574 & 4.31 & $-40,-36,52$ & & & \\
\hline
\end{tabular}

${ }^{a}$ MNI coordinates reflect cluster-center voxels. T-values reflect a statistical threshold of $p<0.01$. Activation clusters survived cluster-threshold correction for multiple comparisons to $p<0.01$ with a minimum cluster size of 144 .

in the hippocampus then activate based on the highest associated reward signal to guide behavior toward the goal location. Our study supports this model by demonstrating that the posterior hippocampus was responsive to the shortest linear distance between participants' current location and the goal location from moment-to-moment as they navigate through the environment. These results provide a novel demonstration that actively coding proximity to a goal location during ground-level navigation in the absence of landmarks recruits the posterior hippocampus.

Recent animal models have suggested that a small portion of cells in the hippocampus may have temporally tuned patterns of activity in addition to spatially specific behavior (Pastalkova et al., 2008; MacDonald et al., 2011; Kraus et al., 2013). These "time cells" may represent a fundamental role of the hippocampus in providing an internal representation of elapsed time, supporting memory for the timing of discrete events. In the current study, participants used self-motion cues to track distance to an encoded goal location. Because time and distance are fundamentally linked during navigation, activations in the hippocampus correlated with proximity to the goal location may, in part, represent cells sensitive to elapsed time. The current task was not specifically designed to separate distance and time in the analyses, so results of our parametric analyses could reflect influences of both time and distance traveled in human navigation. Yet, some models that track distance can be modified to track time elapsed (Hasselmo and Stern, 2013). To further explore this possibility, we modeled two separate analyses to track proximity to the goal location and progression of time across the navigation phase. Our results indicate that activity in posterior left hippocampus, which significantly tracked distance to the goal, was also correlated with time across the FPP navigation phase; however, direct comparison of parameter estimates extracted from our distance to goal and time analyses demonstrates a significantly stronger modula- tion of activity by distance than time in the left hippocampus. Furthermore, activity in the posterior right hippocampus significantly tracked distance to goal but not the progression of time. Together, our results suggest that, during FPP navigation, tracking distance to a goal location has a significant impact on bilateral signal in the hippocampus.

\section{Navigation requiring path integration mechanisms update perceived location and orientation toward a goal location} FPP navigation recruits the hippocampus

The hippocampus may support path integration (Wolbers et al., 2007), which is a mechanism for tracking distance and orientation using self-motion cues. In rodents, persistent spiking of head direction cells, which represent the direction and speed of a trajectory, are thought to update grid cell responses and, thus, update hippocampal place cell activity, giving more accurate knowledge of location in the environment (Burgess et al., 2007; Hasselmo, 2008; 2009). Animal models indicate that a convergence of self-motion and external cues in the hippocampus is essential for path integration and spatial memory processes (Leutgeb et al., 2000). These studies suggest that the hippocampus has a sustained role supporting successful navigation in the absence of landmarks, where there is an increasing reliance on self-motion cues.

The present study targets processes related to integrating survey-level spatial information with ground-level active navigation based on optic flow through simple repeating geometric features. Consistent with its theorized role in path integration, the hippocampus was more active for the navigation phase for successful FPP than successful Survey perspective trials. Hippocampal recruitment for successful FPP navigation is consistent with a framework in which self-motion cues from optic flow support hippocampal position computations. Behavioral studies 
Table 4. Brain regions exhibiting significant activity from paired $t$ test during the map presentation phase ${ }^{a}$

\begin{tabular}{|c|c|c|c|c|c|c|c|}
\hline \multirow{2}{*}{ Contrast } & \multirow[b]{2}{*}{ Area } & \multirow[b]{2}{*}{$K_{E}$} & \multicolumn{2}{|l|}{ Left } & \multirow[b]{2}{*}{$K_{E}$} & \multicolumn{2}{|l|}{ Right } \\
\hline & & & $\mathrm{T}$ & MNI $x, y, z$ & & $\mathrm{~T}$ & MNI $x, y, z$ \\
\hline \multirow[t]{14}{*}{ FPP > Survey } & Hippocampus (tail) & 18,925 & 3.85 & $-30,-38,-4$ & 18,925 & 2.92 & $22,-38,4$ \\
\hline & Hippocampus (body) & & 3.38 & $-22,-30,-12$ & & & \\
\hline & Retrosplenial cortex & & 2.69 & $-10,-46,2$ & & 3.90 & $10,-42,0$ \\
\hline & Precuneus & & 5.60 & $-6,-62,62$ & & 4.12 & $8,-60,56$ \\
\hline & Superior parietal lobule & & 5.34 & $-28,-86,34$ & & 3.87 & $18,-82,44$ \\
\hline & Supramarginal gyrus & & 4.80 & $-46,-46,46$ & & 3.60 & $48,-44,54$ \\
\hline & Parahippocampal cortex & & 3.65 & $-22,-44,-12$ & & 2.77 & $22,-44,-12$ \\
\hline & Dorsal lateral prefrontal cortex & 297 & 5.99 & $-32,40,36$ & & 4.16 & $32,44,36$ \\
\hline & Superior frontal gyrus & & & & & 5.40 & $30,2,68$ \\
\hline & Caudate (dorsal) & 181 & 3.00 & $-16,-2,24$ & & 2.65 & $16,2,22$ \\
\hline & Cuneus & 18,925 & 3.55 & $-2,-88,26$ & & 5.01 & $4,-88,20$ \\
\hline & Angular gyrus & & 3.91 & $-40,-76,22$ & & 4.36 & $34,-74,16$ \\
\hline & Lingual gyrus & & 3.78 & $-18,-58,-2$ & & 3.47 & $10,-62,4$ \\
\hline & Cerebellum & 255 & 5.95 & $-38,-44,-30$ & & 4.96 & $18,-48,-22$ \\
\hline \multirow[t]{13}{*}{ TPP $>$ Survey } & Retrosplenial cortex & 6107 & 2.99 & $-10,-44,2$ & 6107 & 2.88 & $6,-46,8$ \\
\hline & Precuneus & & 3.83 & $-12,-70,50$ & & 3.32 & $22,-68,58$ \\
\hline & Superior parietal lobule & & 4.31 & $-36,-60,58$ & & 5.55 & $34,-60,44$ \\
\hline & Supramarginal gyrus & & 3.52 & $-42,-44,44$ & & 5.28 & $52,-40,46$ \\
\hline & Parahippocampal cortex & & 3.02 & $-20,-40,-12$ & & & \\
\hline & Medial prefrontal cortex (dorsal) & 570 & 4.41 & $-4,30,34$ & 570 & 3.86 & $4,18,42$ \\
\hline & Dorsal lateral prefrontal cortex & 829 & 4.11 & $-52,8,38$ & 2694 & 5.21 & $50,16,22$ \\
\hline & Caudate (dorsal) & 289 & 2.72 & $-14,2,18$ & & 2.75 & $16,4,18$ \\
\hline & Angular gyrus & 6107 & 4.67 & $-30,-82,36$ & 6107 & 3.08 & $34,-72,42$ \\
\hline & Cuneus & & 3.49 & $-18,-96,22$ & & 3.64 & $4,-90,20$ \\
\hline & Cingulate gyrus & 231 & 3.47 & $-2,4,34$ & 231 & 4.65 & $6,12,26$ \\
\hline & Lateral occipital gyrus & & & & 204 & 4.4 & $46,-58,-18$ \\
\hline & Insula & 315 & 4.35 & $-32,16,-2$ & 596 & 5.58 & $40,20,-8$ \\
\hline \multirow[t]{6}{*}{$\mathrm{FPP}>\mathrm{TPP}$} & Retrosplenial cortex & 4970 & 3.86 & $-14,-44,-6$ & & & \\
\hline & Parahippocampal cortex & & & & 4970 & 2.70 & $28,-44,-6$ \\
\hline & Cuneus & & 6.91 & $-10,-82,-32$ & & 8.24 & $16,-78,30$ \\
\hline & Lingual gyrus & & 4.90 & $-12,-68,2$ & & 5.58 & $10,-62,8$ \\
\hline & Lateral occipital gyrus & & & & & 4.00 & $48,-68,16$ \\
\hline & Postcentral gyrus & 556 & 3.32 & $-4,-16,56$ & 556 & 3.47 & $8,-26,54$ \\
\hline $\mathrm{TPP}>\mathrm{FPP}$ & No significant activations & & & & & & \\
\hline
\end{tabular}

${ }^{a} \mathrm{MNI}$ coordinates reflect cluster-center voxels. T-values reflect a statistical threshold of $p<0.01$. Activation clusters survived cluster-threshold correction for multiple comparisons to $p<0.01$ with a minimum cluster size of 144 .
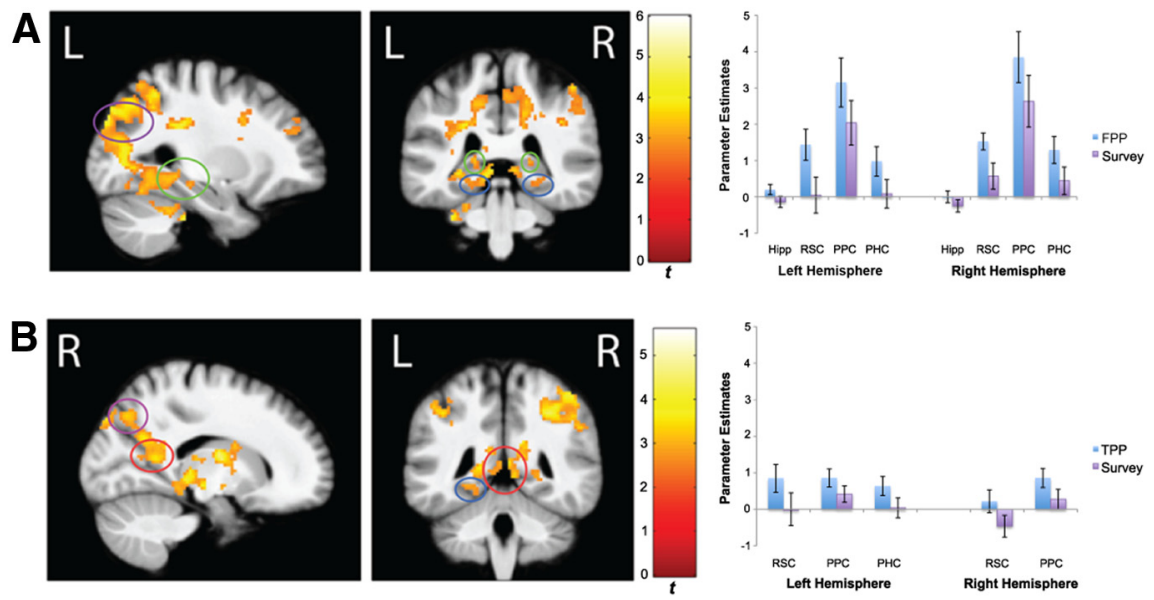

Figure 6. Activations for map presentation trial phase. Both whole-brain analyses images have a statistical threshold of $p<0.01$ corrected for multiple comparisons with a voxel extent of 144. Green circles represent hippocampal (Hipp) activations; red circles represent retrosplenial cortex (RSC) activations; purple circles represent posterior parietal cortex (PPC) activations; blue circles represent parahippocampal cortex (PHC) activations. A, Whole-brain image of activity significantly greater for map presentation phase for subsequent successful FPP navigation against map presentation activation for successive Survey navigation (FPP $>$ Survey). Parameter estimate extractions from ROIs are plotted on the right. Error bars indicate SEM. $\boldsymbol{B}$, Whole-brain image of activity significantly greater for map presentation phase for subsequent successful TPP navigation against map presentation activation for successive Survey navigation (TPP > Survey). Parameter estimate extractions from ROIs are plotted on the right. Error bars indicate SEM. of patients with hippocampal lesions have generally not supported the necessity of the hippocampus for path integration tasks (Shrager et al., 2008; Kim et al., 2013). In contrast, a recent fMRI study of path integration in healthy, young adults has shown that hippocampal activation correlates with angular accuracy in a triangle completion task, wherein participants indicated the direction from their current location back to their start location (Wolbers et al., 2007). In the present study, participants did not need to track their relationship to the start location during FPP navigation; however, path integration may be essential to track current position relative to the goal location based on spatial information encoded at the map presentation.

\section{Regions commonly recruited for FPP and} TPP navigation

In the present study, successful navigation to a goal location from the FPP and TPP relied on self-motion cues to update orientation toward a goal location. During 
FPP and TPP navigation, the retrosplenial cortex and posterior parietal cortex were commonly recruited. The retrosplenial cortex and posterior parietal cortex have been associated with landmark-based navigation (Hartley et al., 2003; Rosenbaum et al., 2004; Spiers and Maguire, 2006; Byrne et al., 2007). In our study, the common recruitment of these brain regions in the absence of landmarks suggests that they play a more basic role in spatial mapping and orientation through path integration. Previous studies suggest the retrosplenial cortex integrates routebased spatial information with self-motion cues (Wolbers and Buchel, 2005) to orient and direct movement to a goal location (Epstein, 2008; Baumann et al., 2010). Data from Spiers and Maguire (2006) demonstrated that posterior parietal cortex was recruited during active navigation to a goal, suggesting a role in the coding and monitoring of response-based spatial information concerning distant locations. Together, our results indicate that posterior parietal cortex activity may support the integration of planned route actions with the spatial relationship between current location and orientation toward the goal location, as represented by the retrosplenial cortex.

Interestingly, the retrosplenial cortex and parahippocampal cortex were commonly recruited for FPP and TPP navigation, yet contrasts of FPP and TPP navigation phases revealed a dissociation in relative activation for navigation in the two perspectives. Navigational demands in the FPP required additional recruitment of the retrosplenial cortex, and additional demands on the parahippocampal cortex were necessary during navigation in the TPP. FPP navigation may have required additional recruitment of the retrosplenial cortex to assist in the integration of selfmotion cues with distance and direction toward goal locations. The parahippocampal cortex may have been recruited to process the changing spatial layout of the scene during TPP navigation. Although the visual input in these perspectives is different, it is also an inherent part of our task design. We think that our contrasts primarily reflect the strategy differences necessary to navigate using self-motion cues in FPP and process changing spatial layout in TPP.

\section{Encoding of large-scale environment required for goal- directed navigation}

Recent studies show spiking activity in the rat hippocampus during sharp wave ripples representing the trajectory the rat subsequently follows from its current location to a known goal location (Pfeiffer and Foster, 2013). Human neuroimaging studies suggest that regions within the MTL, including the hippocampus and parahippocampal cortex, may be important for navigating, and learning to navigate, environments from a ground-level perspective (Hartley et al., 2003; Wolbers and Buchel, 2005; Brown et al., 2010; Weniger et al., 2010; Brown and Stern, 2013). Our experiment characterizes an important facet of the MTL's role in active navigation by demonstrating recruitment of MTL regions during survey-level encoding when the encoded spatial representations were required for successful ground-level navigation. In particular, when navigation was tested in the FPP, bilateral hippocampal activation at map encoding related to successful navigation to the goal location. Our current study demonstrates that encoding of distance and directional measures required for successful FPP navigation recruited the bilateral hippocampus in humans.

\section{References}

Aguirre GK, D’Esposito M (1999) Topographical disorientation: a synthesis and taxonomy. Brain 122:1613-1628. CrossRef Medline

Andersson JL, Hutton C, Ashburner J, Turner R, Friston K (2001) Modeling geometric deformations in EPI time series. Neuroimage 13:903-919. CrossRef Medline

Ashburner J (2007) A fast diffeomorphic image registration algorithm. Neuroimage 38:95-113. CrossRef Medline

Baumann O, Mattingley JB (2010) Medial parietal cortex encodes perceived heading direction in humans. J Neurosci 30:12897-12901. CrossRef Medline

Baumann O, Chan E, Mattingley JB (2010) Dissociable neural circuits for encoding and retrieval of object locations during active navigation in humans. Neuroimage 49:2816-2825. CrossRef Medline

Brown TI, Stern CE (2013) Contributions of medial temporal lobe and striatal memory systems to learning and retrieving overlapping spatial memories. Cereb Cortex. Advance online publication. Retrieved Feb. 28, 2013. doi: 10.1093/cercor/bht041. CrossRef Medline

Brown TI, Ross RS, Keller JB, Hasselmo ME, Stern CE (2010) Which way was I going? Contextual retrieval supports the disambiguation of well learned overlapping navigational routes. J Neurosci 30:7414-7422. CrossRef Medline

Burgess N, Barry C, O'Keefe J (2007) An oscillatory interference model of grid cell firing. Hippocampus 17:801-812. CrossRef Medline

Byrne P, Becker S, Burgess N (2007) Remembering the past and imagining the future: a neural model of spatial memory and imagery. Psychol Rev 114:340-375. CrossRef Medline

Dupret D, O'Neill J, Pleydell-Bouverie B, Csicsvari J (2010) The reorganization and reactivation of hippocampal maps predict spatial memory performance. Nat Neurosci 13:995-1002. CrossRef Medline

Ekstrom AD, Bookheimer SY (2007) Spatial and temporal episodic memory retrieval recruits dissociable functional networks in the human brain. Learn Mem 14:645-654. CrossRef Medline

Ekstrom AD, Kahana MJ, Caplan JB, Fields TA, Isham EA, Newman EL, Fried I (2003) Cellular networks underlying human spatial navigation. Nature 425:184-188. CrossRef Medline

Epstein RA (2008) Parahippocampal and retrosplenial contributions to human spatial navigation. Trends Cogn Sci 12:388-396. CrossRef Medline

Epstein RA, Parker WE, Feiler AM (2007) Where am I now? Distinct roles for parahippocampal and retrosplenial cortices in place recognition. J Neurosci 27:6141-6149. CrossRef Medline

Erdem UM, Hasselmo M (2012) A goal-directed spatial navigation model using forward trajectory planning based on grid cells. Eur J Neurosci 35:916-931. CrossRef Medline

Fanselow MS, Dong HW (2010) Are the dorsal and ventral hippocampus functionally distinct structures? Neuron 65:7-19. CrossRef Medline

Ferbinteanu J, McDonald RJ (2001) Dorsal/ventral hippocampus, fornix, and conditioned place preference. Hippocampus 11:187-200. CrossRef Medline

Foster DJ, Morris RG, Dayan P (2000) A model of hippocampally dependent navigation, using the temporal difference learning rule. Hippocampus 10:1-16. CrossRef Medline

Hartley T, Maguire EA, Spiers HJ, Burgess N (2003) The well-worn route and the path less traveled: distinct neural bases of route following and wayfinding in humans. Neuron 37:877-888. CrossRef Medline

Hasselmo ME (2008) Temporally structured replay of neural activity in a model of entorhinal cortex, hippocampus and postsubiculum. Eur J Neurosci 28:1301-1315. CrossRef Medline

Hasselmo ME (2009) A model of episodic memory: mental time travel along encoded trajectories using grid cells. Neurobiol Learn Mem 92: 559-573. CrossRef Medline

Hasselmo ME, Stern CE (2013) Theta rhythm and the encoding and retrieval of space and time. Neuroimage. Advance online publication. Retrieved June 15, 2013. doi: 10.1016/j.neuroimage.2013.06.022. CrossRef Medline

Johnson A, Redish AD (2007) Neural ensembles in CA3 transiently encode paths forward of the animal at a decision point. J Neurosci 27:1217612189. CrossRef Medline

Kim S, Sapiurka M, Clark RE, Squire LR (2013) Contrasting effects of path integration after hippocampal damage in humans and rats. Proc Natl Acad Sci U S A 110:4732-4737. CrossRef Medline

Kraus BJ, Robinson RJ 2nd, White JA, Eichenbaum H, Hasselmo ME (2013) Hippocampal "time cells": time versus path integration. Neuron 78: 1090-1101. CrossRef Medline

Leutgeb S, Ragozzino KE, Mizumori SJ (2000) Convergence of head direc- 
tion and place information in the CA1 region of hippocampus. Neuroscience 100:11-19. CrossRef Medline

MacDonald CJ, Lepage KQ, Eden UT, Eichenbaum H (2011) Hippocampal "time cells" beidge the gap in memory for discontiguous events. Neuron 71:737-749. CrossRef Medline

Maldjian JA, Laurienti PJ, Kraft RA, Burdette JH (2003) An automated method for neuroanatomic and cytoarchitectonic atlas-based interrogation of fMRI data sets. Neuroimage 19:1233-1239. CrossRef Medline

Moser MB, Moser EI (1998) Functional differentiation in the hippocampus. Hippocampus 8:608-619. CrossRef Medline

O'Keefe J, Dostrovsky J (1971) The hippocampus as a spatial map: preliminary evidence from unit activity in the freely-moving rat. Brain Res 34: 171-175. CrossRef Medline

Pastalkova E, Itskov V, Amarasingham A, Buzsáki G (2008) Internally generated cell assembly sequences in the rat hippocampus. Science 321:13221327. CrossRef Medline

Pfeiffer BE, Foster DJ (2013) Hippocampal place-cell sequences depict future paths to remembered goals. Nature 497:74-79. CrossRef Medline

Rodriguez PF (2010) Human navigation that requires calculating heading vectors recruits parietal cortex in a virtual and visually sparse water maze task in fMRI. Behav Neurosci 124:532-540. CrossRef Medline

Rosenbaum RS, Ziegler M, Winocur G, Grady CL, Moscovitch M (2004) "I have often walked down this street before": fMRI studies on the hippocampus and other structures during mental navigation of an old environment. Hippocampus 14:826-835. CrossRef Medline

Royer S, Sirota A, Patel J, Buzsáki G (2010) Distinct representations and theta dynamics in dorsal and ventral hippocampus. J Neurosci 30:17771787. CrossRef Medline

Sato N, Sakata H, Tanaka YL, Taira M (2006) Navigation-associated medial parietal neurons in monkeys. Proc Natl Acad Sci U S A 103:17001-17006. CrossRef Medline

Save E, Poucet B (2009) Role of the parietal cortex in long-term representation of spatial information in the rat. Neurobiol Learn Mem 91:172-178. CrossRef Medline
Shrager Y, Kirwan CB, Squire LR (2008) Neural basis of the cognitive map: path integration does not require the hippocampus or medial entorhinal cortex. Proc Natl Acad Sci U S A 105:12034-12038. CrossRef Medline

Spiers HJ, Maguire EA (2006) Thoughts, behavior, and brain dynamics during navigation in the real world. Neuroimage 31:1826-1840. CrossRef Medline

Spiers HJ, Maguire EA (2007) A navigational guidance system in the human brain. Hippocampus 17:618-626. CrossRef Medline

Vann SD, Aggleton JP, Maguire EA (2009) What does the retrosplenial cortex do? Nat Rev Neurosci 10:792-802. CrossRef Medline

Viard A, Doeller CF, Hartley T, Bird CM, Burgess N (2011) Anterior hippocampus and goal-directed spatial decision making. J Neurosci 31:46134621. CrossRef Medline

Weniger G, Siemerkus J, Schmidt-Samoa C, Mehlitz M, Baudewig J, Dechent P, Irle E (2010) The human parahippocampal cortex subserves egocentric spatial learning during navigation in a virtual maze. Neurobiol Learn Mem 93:46-55. CrossRef Medline

Whitlock JR, Pfuhl G, Dagslott N, Moser MB, Moser EI (2012) Functional split between parietal and entorhinal cortices in the rat. Neuron 73:789802. CrossRef Medline

Wolbers T, Büchel C (2005) Dissociable retrosplenial and hippocampal contributions to successful formation of survey representations. J Neurosci 25:3333-3340. CrossRef Medline

Wolbers T, Wiener JM, Mallot HA, Büchel C (2007) Differential recruitment of the hippocampus, medial prefrontal cortex, and the human motion complex during path integration in humans. J Neurosci 27: 9408-9416. CrossRef Medline

Xu J, Evensmoen HR, Lehn H, Pintzka CW, Håberg AK (2010) Persistent posterior and transient anterior medial temporal lobe activity during navigation. Neuroimage 52:1654-1666. CrossRef Medline

Zhang H, Ekstrom A (2013) Human neural systems underlying rigid and flexible forms of allocentric spatial representation. Hum Brain Mapp 34: 1070-1087. CrossRef Medline 